\title{
Effect of Evaporated Ethyl Pyruvate on Reducing Salmonella Enteritidis in Raw Chicken Meat
}

http://dx.doi.org/10.1590/1806-9061-2018-0781

Euthor(s)

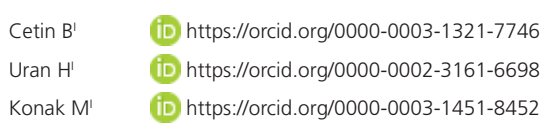

Kırklareli University, Faculty of Engineering, Dept of Food Engineering, Kayali Campus, Kırklareli, Turkey.

\section{-Mail Address}

Corresponding author e-mail address Bayram Cetin

Kırklareli University, Faculty of Engineering Department of Food Engineering, Kayali

Campus, Kırklareli, Turkey.

Phone: +902882140514

Email: bayram.cetin@klu.edu.tr

\section{ABSTRACT}

In this study, the effect of evaporated ethyl pyruvate (EP) was evaluated for the decontamination of Salmonella Enteritidis on chicken leg meat as a safe alternative to antimicrobial agent. Also, total aerobic mesophilic bacteria (TAMB), Enterobacteriaceae, Escherichia coli and yeast-mold counts samples were investigated. Subsequently, the samples were injected with 0, 42, 105 and $420 \mathrm{mg}$ evaporated EP/L air to the paper filter attached to the container cover and stored at $+4{ }^{\circ} \mathrm{C}$ for 10 days. According to the results, $42 \mathrm{mg}$ EP/L concentration did not cause a significant decrease in Salmonella Enteritidis count ( $p>0.05$ ). However, it was determined that 105 and $420 \mathrm{mg}$ EP/L treatments reduced the number of Salmonella Enteritidis by more than 1 and 2 log, respectively. EP application also significantly influenced the number of TAMB, Enterobacteriaceae and yeast-mold. These results indicate that $E P$ is an effective antimicrobial that could be used to enhance the safety of chicken meat.

\section{INTRODUCTION}

The poultry meat has become the second crop after beef and has proven to be an alternative to the closure of the protein opening. Chicken meat is more economical and show lower levels of cholesterol when compared to other poultry meats, so chicken meat consumption has increased (Caki, 2007). Poultry meats are highly perishable to bacterial contaminants due to large amounts of variable nutrients, a high water activity (aw) and a higher final $\mathrm{pH}$ limiting the shelf-life of the product (Cantalejo et al., 2016). Salmonella sp., Listeria monocytogenes and Campylobacter sp., which cause diseases in man, can be found on poultry carcasses and in poultry processing plants (Nierop et al., 2005). Mostly, taste and smell modifications haven't occurred in Salmonella grown meats.

Salmonella is the most commonly reported cause of foodborne disease among bacterial infections. It is estimated that about 94 million cases of gastroenteritis due to Salmonella species occur annually worldwide, leading to 155,000 deaths every year. Among Salmonella species, Salmonella Enteritidis that cause human nontyphoidal salmonellosis is isolated predominantly from poultry. In recent years, $S$. Enteritidis has been reported as a major causative agent of foodborne gastroenteritis in humans (Asif et al., 2016). The contamination of Salmonella sp. may occur throughout the chicken production chain and processing steps. Head pulling and evisceration are considered potential risk factors that contribute to high incidence of Salmonella sp. in chicken carcasses (Hungaro et al., 2013).

There are some physical interventions that have been used to reduce the bacterial contamination on chicken carcasses. They include 
mainly, water-based and steam treatments, irradiation, ultrasound, high hydrostatic pressure processing and pulsed electric field processing. Amongst these physical methods, water-based and steam treatments have been used frequently for the decontamination of poultry carcasses, and yielded reductions for various bacterial species in the range from 0.9 to 2.1 and 2.3 to 3.8 orders of magnitude, respectively (Loretz et al., 2009).

Changes in the appearance and quality of carcasses, the need for equipment and operator training, and consumer rejection constitute major limitations for the application of physical methods. Also, during slaughter and processing, chemicals such as trisodium phosphate, chlorine-based compounds and organic acids are used in some countries to assist in the reduction of microorganisms in poultry carcasses (Del Río et al., 2007). For example, after treated with trisodium phosphate, chlorine-based compounds, and lactic or acetic acid Salmonella contamination has been reduced by $0.6-2.3,0.9-1.1$, and $0.8-2.2 \log$ units, respectively. (Buncic \& Sofos, 2011). But, the effectiveness of chemical decontamination methods may remain limited as they may not have access to the microbial pathogens which penetrated inner sites of chicken skin. Gaseous phase and volatile antimicrobials can reach the hidden areas of products and thus, have the potential to eliminate disadvantages of such decontamination processes. Several antimicrobials in the gaseous or vapor phase, including chlorine, ozone, allyl isothiocynate (AIT), methyl isothiocyanate and essential oils have been used to inactivate pathogens on fresh produce (Durak et al., 2012).

Ethyl pyruvate (EP) is one of the volatile antimicrobials and is a lipophilic pyruvate derivative safer and more stable than pyruvate. It has antiinflammatory and antioxidative effects. It is currently used as a food additive and flavouring agent known as a therapeutic agent. EP treatment is an emerging nonthermal technology for reducing microbial load on the surface of fresh and processed foods. EP can reach and penetrate the sites that microorganisms can be hidden because of that it is so volatile (Chen et al., 2017; Tornuk \& Durak, 2015). EP has antimicrobial properties against microorganisms with clinical relevant resistance (bacteria, fungi, moulds, parasites). Furthermore, EP has shown reduced anti-microbial activities against symbiotic microorganisms such as Lactobacillus species (Debebe et al., 2016). EP is classified as GRAS (generally recognized as safe) by the U.S. Food and Drug Administration under certain conditions. However, antimicrobial activity of EP against pathogens and its antimicrobial activity in broiler chickens have not been previously reported. In the present study, the antimicrobial properties of vaporized EP on Salmonella Enteritidis ATCC 13076 and on sensorial and other microbiological parameters such as total aerobic mesophilic bacteria, Enterobacteriaceae, Escherichia coli and yeast-mold counts in chicken leg meat were investigated.

\section{MATERIALS AND METHODS}

\section{Bacterial cultures}

Salmonella (S.) Enteritidis ATCC 13076 was used for decontamination of chicken leg meat samples in this research. The bacterial culture was provided from Yildiz Technical University Food Engineering Department, Istanbul, Turkey. The bacterial culture was stored on agar slants at $4^{\circ} \mathrm{C}$. Overnight cultures were prepared freshly for each experiment by incubation at $37^{\circ} \mathrm{C}$ for $16 \mathrm{~h}$ in tryptone soya broth (TSB, Oxoid, asingstoke, UK). The inoculum cocktail (1:1000 dilution, $10^{8}$ colony forming units (CFU)/mL) was prepared by mixing $3 \mathrm{ml}$ bacterial suspension in $3 \mathrm{~L}$ of $0.1 \%$ peptone water. Fresh activated bacterial cells concentration was approximately adjusted to $4 \mathrm{log}$ CFU/mL for stock inoculum solution (Durak et al., 2012).

\section{Preparation of Meat Samples}

Chicken leg meats were purchased from a local market in Kirklareli, Turkey and all samples were swiftly taken to the laboratory. Chicken leg meat samples were contaminated with inoculum culture for 10 minutes in the immersion water as initial numbers $10^{4} \mathrm{CFU} / \mathrm{g}$ (Durak et al., 2012) before the experiment. For the experiment, chicken leg meat samples were divided into 4 groups (0 (control), 42, 105 and 420 $\mathrm{mg} / \mathrm{L}$ vaporized $\mathrm{EP}$ ) with the amounts of $200 \mathrm{~g}$ for each sample.

\section{Application of EP}

A $200 \mathrm{~g}$ sample of inoculated chicken leg meat was placed in a 1-L closed-lid food container $(18.00 \mathrm{~cm} \times 25.50 \mathrm{~cm} \times 9.00 \mathrm{~cm}$, Bora Plastic, Istanbul, Turkey). Application of EP (98\% purity; Sigma Aldrich, St. Louis, MO) was performed according to the method described by Durak et al. (2012) with slight modifications. To generate EP vapor overtime, 105, 260 and 1,050 $\mu \mathrm{L}$ (equivalent to 42, 105, and 420 $\mathrm{mg} / \mathrm{L}$ air) of EP was deposited onto Kim Wipes tissues (Kimberly- Clark, Rose well, GA) in the 1-liter food containers. After sealing the containers, the samples 
were stored at refrigerator temperature $\left(+4^{\circ} \mathrm{C}\right)$ for 10 days. The control sample did not contain EP in the sealed containers. The samples stored at $4^{\circ} \mathrm{C}$ were tested on days $0,3,6,8$, and 10. All EP experiments and analyses of samples were performed in duplicate format.

\section{ANALYSES}

\section{Microbiological analyses}

Microbiological analysis conducted in this study included the determination of S. Enteritidis ATCC 13076, at the same time Enterobacterieaceae, Escherichia (E.) coli, yeast-mold, and TAMB counts using conventional cultural methods. For this objective, the sample $(10 \mathrm{~g})$ was transferred to $90 \mathrm{~mL} 0.1 \%$ peptone water (Oxoid, Basingstoke, Hampshire, England) and homogenized with Stomacher Lab-Blender 400 (Seward Medical, London, UK). Appropriate 10-fold dilutions of the samples were prepared in sterile peptone water. Appropriate dilutions were inoculated on petri dishes with Xylose Lysine Deoxycholate (XLD) agar (Merck, Darmstadt, Germany) or Brilliant-green Phenolred Lactose Sucrose agar (Merck) for enumeration of S. Enteritidis ATCC 13076 using spread-plate technique. Then the plates were incubated for $24 \mathrm{~h}$ at $37^{\circ} \mathrm{C}$. After incubation; some biochemical tests were made to identify Salmonella species. Specific colonies were counted following the identification (Mahmoud \& Linton, 2008). The enumeration of Enterobacteriaceae was performed by Violet Red Bile Dextrose Agar (VRBDA, Oxoid, Basingstoke, Hampshire, England) by using pour plating method and plates were incubated at $37{ }^{\circ} \mathrm{C}$ for $24 \mathrm{~h}$ according to the standard procedure (TS ISO 21528-2:2012). Numbers of beta-glucuronidase-positive $E$. coli were quantified on Tryptone Bile X-Glucuronide (TBX) Agar (Oxoid), followed by $4 \mathrm{~h}$ and subsequent $24 \mathrm{~h}$ of incubation at $30^{\circ} \mathrm{C}$ and $44^{\circ} \mathrm{C}$, respectively, according to the ISO 16649-2:2001. Total yeast-mold count was determined in Rose Bengal Chloramphenicol Agar (Oxoid) by using surface plating method and plates were incubated at $25^{\circ} \mathrm{C}$ for 5-7 days (ISO 21527-2:2008). Total count of aerobic mesophilic bacteria (TAMB) in samples was determined by using pour plate method on plate count agar (Oxoid) and incubating the plates at a temperature of $30^{\circ} \mathrm{C}$ for $24-48 \mathrm{~h}$. All plates were incubated under aerobic conditions (ISO 4833-2:2013). The results were converted to logarithmic values. The analyses were performed in duplicate and the results were expressed as CFU/g.

\section{Growth Inhibition Level}

In addition to the determination of bacterial counts, growth inhibition levels (GIL) of S. Enteritidis ATCC 13076 caused by the effect of vaporized EP were calculated using the following equation (Eq. 1) as applied by Sagdic (2003):

$$
G I L(\%)=\frac{(P C-P r)}{P C} \times 100
$$

Where $P_{C}$ and $P_{T}$ are the microbial populations of the control and EP-treated samples at a certain time, respectively.

\section{Visual Evaluation}

Control and EP-treated chicken leg samples after inoculation S. Enteritidis ATCC 13076 were visually evaluated during the storage period for color, odor, texture, and overall quality (acceptability) by a semitrained panel of 10 individuals and rated with a 7-point hedonic scale, where 1 represented 'dislike extremely' and 7 represented 'like extremely'. Samples were considered acceptable when their mean scores were above 4 (neither like nor dislike) (Tornuk \& Durak, 2015; Durak et al., 2012). The degree of decay in the samples and their color, odor and viscidity properties were verbally explained by the evaluators (Tornuk \& Durak, 2015).

\section{Statistical analysis}

Analysis of variance (ANOVA) was conducted for each variable measured to investigate the effect of treatments during storage time. After the data were obtained, differences were defined with SPSS 18.0 using Tukey's multiple range tests. Differences were considered significant at $p<0.01$.

\section{RESULTS}

\section{Inactivation of S. Enteritidis ATCC 13076 by Vaporized Ethyl Pruvate}

The inhibitor effect of ethyl pyruvate application at different concentrations on S. Enteritidis throughout the storage period for chicken leg meat samples stored at $4^{\circ} \mathrm{C}$ are shown in Table 1 . The starting population of S. Enteritidis ATCC 13076 prior to EP application in chicken leg meat samples was determined as $4.91 \mathrm{log}$ CFU/g. When compared with the control group $(0 \mathrm{mg}$ $E P / L)$, it was observed that the lowest EP application (42 mg EP/L) could not provide significant inactivation throughout the storage period ( $p>0.05$ ). However, 105 and $420 \mathrm{mg}$ EP/L applications provided decreases of 1.00 and $1.92 \mathrm{log}$ CFU/g respectively which resulted in a statistically significant difference $(p<0.01)$ (Figure 1). 
Table 1 - Inactivation of S. Enteritidis ATCC 13076 on chicken leg meat by various concentrations of vaporized ethyl pyruvate (EP) at $4^{\circ} \mathrm{C}$ in 10 days ${ }^{1}$.

\begin{tabular}{lccccc}
\hline Number of Salmonella Enteritidis ATCC 13076 (log CFU/g) & \multicolumn{3}{c}{ Storage Time (Day) } \\
\cline { 2 - 6 } Example & 0 & 3 & 6 & 8 & 10 \\
\hline $0 \mathrm{mg} \mathrm{EP/L}$ & $4.40 \pm 0.05^{\mathrm{a}}$ & $4.63 \pm 0.16^{\mathrm{a}}$ & $4.68 \pm 0.04^{\mathrm{a}}$ & $4.87 \pm 0.05^{\mathrm{a}}$ & $4.91 \pm 0.09^{\mathrm{a}}$ \\
\hline $42 \mathrm{mg} \mathrm{EP/L}$ & $4.40 \pm 0.05^{\mathrm{a}}$ & $4.33 \pm 0.05^{\mathrm{a}}$ & $4.64 \pm 0.04^{\mathrm{a}}$ & $4.31 \pm 0.11^{\mathrm{a}}$ & $4.53 \pm 0.01^{\mathrm{a}}$ \\
\hline $105 \mathrm{mg} \mathrm{EP} / \mathrm{L}$ & $4.40 \pm 0.05^{\mathrm{a}}$ & $4.09 \pm 0.21^{\mathrm{ab}}$ & $3.91 \pm 0.03^{\mathrm{b}}$ & $3.85 \pm 0.02^{\mathrm{b}}$ & $3.40 \pm 0.31^{\mathrm{b}}$ \\
\hline $420 \mathrm{mg} \mathrm{EP/L}$ & $4.40 \pm 0.05^{\mathrm{a}}$ & $3.34 \pm 0.37^{\mathrm{b}}$ & $3.11 \pm 0.25^{\mathrm{c}}$ & $3.10 \pm 0.02^{\mathrm{c}}$ & $2.48 \pm 0.00^{\mathrm{c}}$ \\
\hline
\end{tabular}

${ }^{1}$ Values include \pm standard deviation.

Values shown in different letters in the same column are statistically different. ( $p>0.01)$

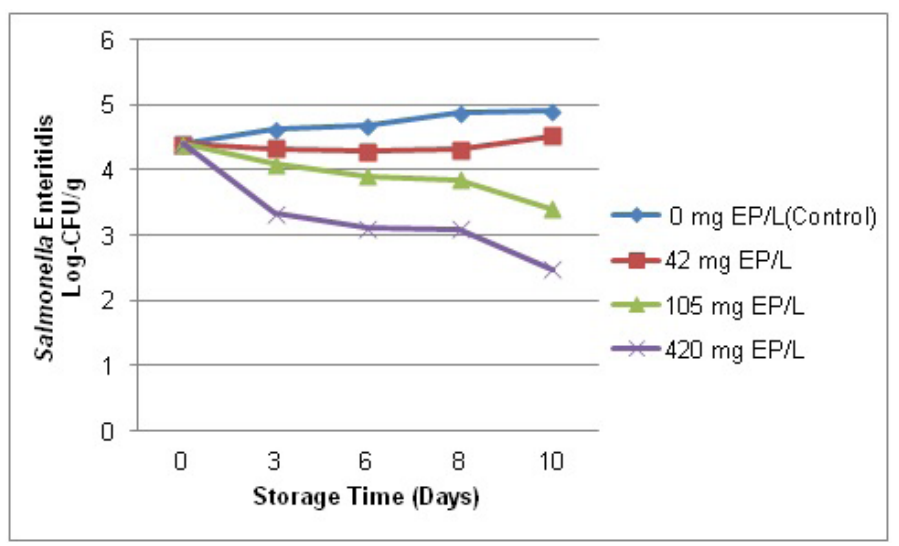

Figure 1 - Changes in the number of $S$. Enteritidis of chicken leg meat during storage period.

Figure 5 shows GILs of S. Enteritidis ATCC 13076 inoculated to chicken leg meat. The concentration dependence of the inhibition levels can be clearly seen from this figure. At low inoculum levels, there were no remarkable differences between the GILs obtained by $42 \mathrm{mg} E P / L$ treatment. On the other hand, 105 or $420 \mathrm{mg} E P / L$ treatments were notably effective as compared with $42 \mathrm{mg}$ EP/L.

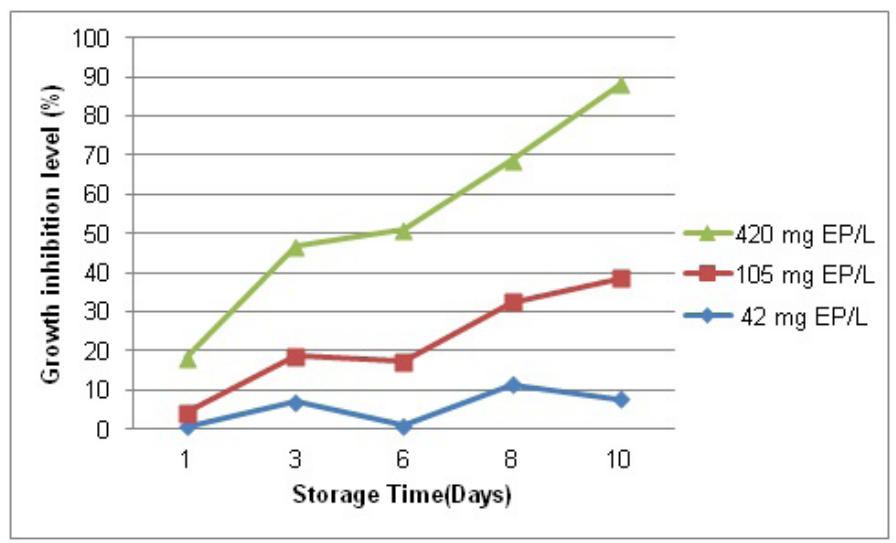

Figure 5 - Growth Inhibition Levels (GIL) of ethyl pyruvate at different concentrations against $S$. Enteritidis on chicken leg meats.

\section{Antimicrobial Activity of EP}

Variousmicrobiologicalanalyses(Enterobacteriaceae, E. coli, TAMB and yeast-mold count) were carried out on chicken leg samples applied with evaporated ethyl pyruvate and stored at $4^{\circ} \mathrm{C}$ for 10 days after which the effects of EP application on these microbiological criteria were examined.

When chicken leg meat samples were examined with regard to $E$. coli count, these group bacteria were determined in the control group samples, however E. coli development was not observed on EP applied samples. The antimicrobial effect of EP application occurred especially on Enterobacteriaceae, TAMB and yeast-mold count. EP application resulted in a statistically significant difference between the samples with regard to Enterobacteriaceae count. Whereas Enterobacteriaceae count in the control group samples was determined as $6.7 \mathrm{log}$ CFU/g on the $10^{\text {th }}$ day of storage; this value was determined as $6.08 ; 3.28$ and $2.09 \log$ CFU/g for 42, 105 and $420 \mathrm{mg} \mathrm{EP} \mathrm{/L} \mathrm{applied}$ sample groups, respectively. A significant reduction was observed in Enterobacteriaceae development depending on the increase in EP concentration in the samples (Figure 2). 105 and $420 \mathrm{mg} \mathrm{EP/L}$ applications prevented Enterobacteriaceae development at a significant level respectively as $3.42 \mathrm{log}$ CFU/g and $4.61 \log$ CFU/g after 10 days of storage $(p<0.01)$.

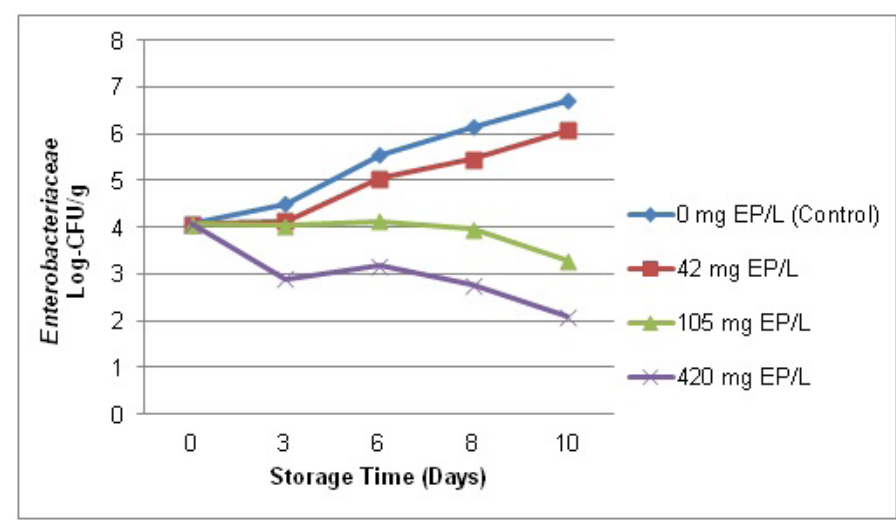

Figure $\mathbf{2}$ - Changes in the number of Enterobacteriaceae of chicken leg meat during storage period.

A statistically significant inhibition in the TAMB counts was also observed in the samples throughout 
the storage period depending on the increase in the EP concentration. Whereas TAMB count was determined as $7.69 \mathrm{log}$ CFU/g on the $10^{\text {th }}$ day of storage for the control group samples; this value was determined as $6.55 ; 5.19$ and $3.76 \mathrm{log}$ CFU/g respectively for 42 , 105 and $420 \mathrm{mg}$ EP/L applied sample groups (Figure 3). In comparison with the control group, $42 \mathrm{mg} / \mathrm{L} \mathrm{EP}$ application resulted in a reduction at a level of 1.14 $\log$ CFU/g, $105 \mathrm{mg}$ EP/L application resulted in a reduction at a level of $2.50 \mathrm{log}$ CFU/g and $420 \mathrm{mg}$ EP/L application resulted in a reduction at a level of $3.93 \mathrm{log}$ $\mathrm{CFU} / \mathrm{g}(p<0.01)$.

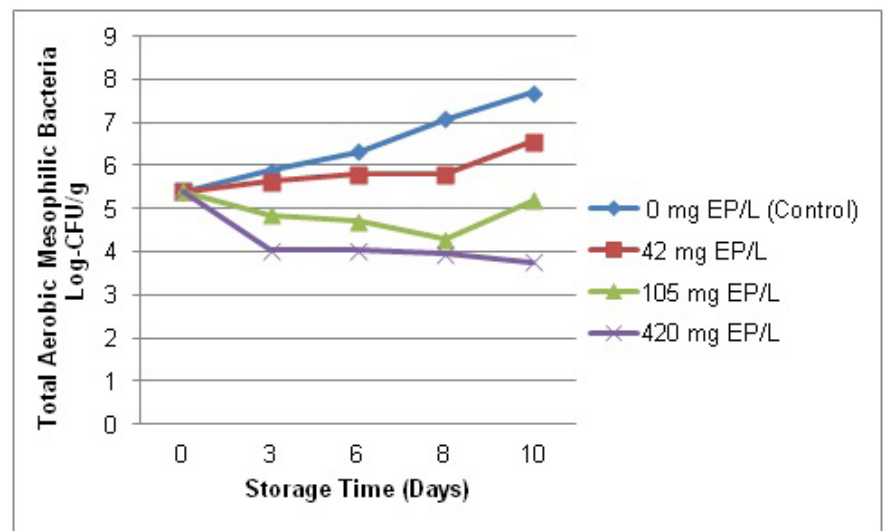

Figure 3 - Changes in the number of total aerobic mesophilic bacteria of chicken leg meat during storage period.

EP application resulted in a significant effect on the yeast-mold count of chicken leg meat samples. Whereas the yeast-mold count of the control group samples was determined as $6.64 \mathrm{log}$ CFU/g on the $10^{\text {th }}$ day of storage; this value on the same day was determined as 5.80; 3.59 and $<0.5 \log$ CFU/g for 42, 105 and $420 \mathrm{mg}$ EP/L applied samples respectively (Figure 4). After 10 days of storage, 42, 105 and $420 \mathrm{mg}$ EP/L applications resulted in decreases of $0.84 \log$ CFU/g; $3.05 \mathrm{log}$ CFU/g and $<6.14 \mathrm{log}$ CFU/g respectively with the highest inhibitor effect on yeast and mold development $(p<0.01)$.

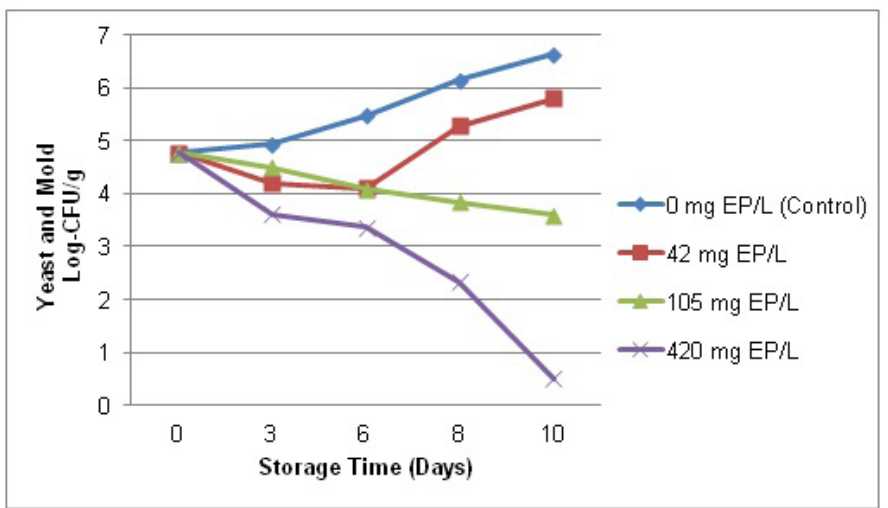

Figure 4 - Changes in the number of yeast-mold of chicken beef samples during storage period

\section{Visual evaluation findings}

Chicken leg meat samples were examined visually with regard to color, odor and stickiness throughout the storage period. Evaluators who carried out the visual evaluation put forth that they felt no difference regarding the odor when the samples of 42 and 105 mg EP/L applications were compared with the control group samples. However, strong EP odor was felt throughout the storage period in $420 \mathrm{mg}$ EP/L applied samples. It was stated that EP applied samples were in better condition in comparison with the control group samples regarding the color. The stickiness levels of the samples yielded similar results and it was emphasized that highest EP applied samples (420 mg $\mathrm{EP} / \mathrm{L}$ ) were determined to be more positive with respect to stickiness.

\section{DISCUSSION}

The decontamination effects on $S$. Enteritidis ATCC 13076 of EP application evaporated at different concentrations in chicken leg meat samples stored at $+4^{\circ} \mathrm{C}$ were examined in this study. In addition, the effects of this application on various microbial and sensory quality properties of the samples were also examined.

The effectiveness of antimicrobials in the vapor phase generally depend on different factors (Nadarajah et al., 2005; Obaidat \& Frank, 2009; Durak et al., 2012). The inhibitor effect of evaporated EP on S. Enteritidis increased depending on EP concentration and storage time. Whereas the lowest concentration of $42 \mathrm{mg}$ EP/L evaporated EP application did not result in a significant inhibition effect on $S$. Enteritidis in chicken leg meat samples; it was observed that evaporated EP applications applied at concentrations of 105 and 420 mg EP/L decreased S. Enteritidis population in chicken leg meat samples faster and continuously throughout the storage period (Figure 1 and 2). Whereas $105 \mathrm{mg}$ $\mathrm{EP} / \mathrm{L}$ application resulted in a decrease of $1 \mathrm{log}$ in the samples at the end of the $10^{\text {th }}$ day, EP applied at a concentration of $420 \mathrm{mg} / \mathrm{L}$ resulted in a decrease of approximately $1.92 \log$ (Table 1). Increase of EP concentration significantly affects $S$. Enteritidis inhibition as well. It is considered that EP application above $105 \mathrm{mg}$ EP/L shall provide significant $S$. Enteritidis inhibition and that it will be able to prevent low levels of contaminations or recontaminations completely.

Microbial inhibition increases with increasing EP concentration and the shelf life of the samples increases. Durak et al. (2012) carried out a study examining E. coli 
0157:H7 decontamination in green onion and spinach samples with EP application as a result of which it was put forth that the lowest EP concentration (42 mg $E P / L)$ resulted in a reduction of 1.7 in green onion and a reduction of $0.9 \mathrm{log}$ CFU/g in spinach at the end of 7 days storage at $4^{\circ} \mathrm{C}$. Researchers observed that under the same conditions, the highest concentration (420 $\mathrm{mg} \mathrm{EP} / \mathrm{L}$ ) resulted in a reduction of above 4.7 in green onion and a reduction of $4.3 \mathrm{log}$ CFU/g in spinach. It was emphasized as a result of the study that this application can be used for improving the freshness and shelf life of the product and for developing an alternative preservation method.

Temperature is among the most important factors with impact on the effectiveness of antimicrobials in the vapor phase and this is indicated in the studies carried out (Obaidat \& Frank, 2009; Durak et al., 2012). Even though significant inhibition $(p<0.01)$ was attained in samples applied with 105 and $420 \mathrm{mg} \mathrm{EP/L}$, results of studies carried out with vegetables (Durak et al., 2012; Tornuk \& Durak, 2015) were below the determined levels. It is thought that quite low $\left(+4^{\circ} \mathrm{C}\right)$ storage temperature and the physical structure of the chicken leg meat samples have been effective on obtaining this result. The effectiveness of EP vapor application also modifies with changes in factors such as container composition and surface voids (Durak et al., 2012).

There are studies on Salmonella inactivation in foods. It has been suggested that plant hydrosols and especially thyme hydrosol have inhibitor effects on $S$. Typhimurium and that they yield a reduction of above $1 \log$ CFU/g in fruits (Tornuk et al., 2011). 5 $\mathrm{mg} / \mathrm{L}$ chlorine dioxide gas application for 2 minutes on lettuce resulted in a decrease of $1.5 \mathrm{log}$ in $\mathrm{S}$. enterica count (Mahmoud \& Linton, 2008). All living cells (5 x $10^{3} \mathrm{CFU}$ ) were eliminated in a study carried out for examining the inhibition of Salmonella Enteritidis in raw chicken meat with carvacrol vapor (Burt et al., 2007). Spray washing of broiler carcasses with Electroloyzed Oxidated (EO) or Sodium Hypochlorite solution (HOCL) resulted in a decrease in Salmonella level by 1.2 and 1.5 log respectively (Northcutt et al., 2007). This new method that was applied for the first time on chicken leg meat samples provides inhibition at the same level or even at a greater level in comparison with the different applications suggested for Salmonella decontamination chicken meat and vegetables. In addition, ease of application is the greatest advantage of this method.

In our study, Enterobacteriaceae, E. coli, yeastmold and TAMB counts of chicken leg meat samples were determined along with $\mathbf{S}$. Enteritidis in addition to examining the effects of EP application on the microbiological quality of the samples. Evaporated EP applied at a concentration of $420 \mathrm{mg}$ EP/L resulted in a decrease by about $4.6 \mathrm{log}$ in the Enterobacteriaceae count of the samples at the end of the $10^{\text {th }}$ day. Whereas E. coli was determined in non-EP applied (control group) samples throughout the storage period, this bacteria could not be determined in samples subject to even the lowest EP concentration (42 mg $E P / L)$. This indicates that $E$. coli is quite sensitive to $E P$ application and that evaporated EP may be applied as a quite effective method in comparison with studies carried out for $E$. coli decontamination (Nadarajah et al., 2005; Han et al., 2000; Obaidat \& Frank 2009; Vurma et al., 2009; Sagdic et al., 2013).

It has been observed in our study that the yeastmold load decreased at a statistically significant level based on EP concentration. EP applied at a concentration of $420 \mathrm{mg} E P / L$ decreased all yeast-mold population below the detection limit $(<0.5 \mathrm{log})$ at the end of the $10^{\text {th }}$ day. EP application provided inhibition at levels of 1.14, 2.5 and $3.93 \mathrm{log}$ from the lowest application level towards the highest level in aerobic colony count at the end of a 10 day storage period. It was observed that the increase in EP in our study provided a statistically significant decrease in the TAMB count of chicken leg meat samples.

There are many studies carried out for examining the effects of antimicrobial effects of vaporizable chemicals on various vegetables (Mahmoud \& Linton, 2008; Obaidat \& Frank, 2009; Bozkurt et al., 2015). For example, Burt et al. (2007) carried out a study in which they managed to remove $S$. Enteritidis in raw meat by applying carvacrol vapor. It was determined during these studies that the chemicals applied in vapor phase have statistically significant inhibitor effect on microorganisms. Being subjected to chlorine vapor for long periods of time may result in skin and respiratory tract irritation and chlorine dioxide may explode at high densities (Beuchat, 1998). Allyl isothiocyanate (AIT) is a strong skin and mucosa membrane irritant (Gosselin et al., 1982) and ozone gas is a strong oxidizer, in addition, it may also result in psychological disorders and corrosion on metal surfaces. Ozone generation technologies are also generally complicated and are expensive for many practical applications (Durak et al., 2012). Consideration for sensory quality of chicken leg meat samples within EP treatments, visual properties of the control and EP-treated samples were examined and no differences were observed regarding the odor 
or visual change in the samples of 42 and $105 \mathrm{mg} E P / \mathrm{L}$ applications. Increasing EP concentration throughout the storage period in $420 \mathrm{mg} E P / L$ applied samples were determined to be lightly positive with respect to stnki. In the previous studies, EP-treated baby spinach and fresh parsley samples had been reported to have generally lower sensorial attributes than those of the control samples after storage (Tornuk \& Durak, 2015, Durak etal. 2012). In this study EP concentrations also decreased clearly over 7 days of storage at $4^{\circ} \mathrm{C}$ to $<5$ $\mathrm{mg} / \mathrm{L}$ in the containers. Similarly, Durak et al. (2012) reported that only trace of concentrations of EP were detectable at the end of the experiment, and thus, the efficacy of EP vapor treatments may vary with changes in such factors as the container composition or surface area.

It was determined as a result of the study that evaporated EP provides reduction in S. Enteritidis count as well as other microbial count in chicken leg meat and that the most ideal application is $105 \mathrm{mg} \mathrm{EP/L}$. In addition to being the first known study examining the antimicrobial activity of EP on S. Enteritidis, this study also supports the EP application as an effective decontamination method. Nevertheless, impact mechanism of the EP has not been fully understood yet and this has been expressed in some studies (Durak et al., 2012). It is thought that evaporated EP which is a new application has significant antimicrobial effect, that can be used easily in many foods with high microbial contamination risks, that it may be used as an alternative to storage methods such as modified atmosphere and that it can be applied for the decontamination of carcasses at the slaughter house following the cutting.

\section{REFERENCES}

Beuchat L R. Surface decontamination of fruits and vegetables eaten raw: a review. WHO 1998; p.1-42. Available from: http://www.who.int/iris/ handle/10665/64435

Debebe T, Krüger M, Huse K, Kacza J, Mühlberg K, König B, Birkenmeier G. Ethyl Pyruvate: An Anti-Microbial Agent that Selectively Targets Pathobionts and Biofilms. Plos One 2016; 11(9):1-19. Available from: DOI:https://doi.org/10.1371/journal.pone.0162919

Burt SA, Fledderman MJ, Haagsman HP, van Knapen F, Veldhuizen EJA. Inhibition of Salmonella enterica serotype Enteritidis on agar and raw chicken by carvacrol vapour. International Journal of Food Microbiology 2007; 119: 346-350. DOl:https://doi.org/10.4315/0362-028X.JFP-12008

Buncic S, Sofos J. Interventions to control Salmonella contamination during poultry, cattle and pig slaughter. Food Research International 2011; 45:641-655. DOI:https://doi.org/10.1016/j.foodres.2011.10.018
Caki SS. The Place and Situation of Poultry Industry in Turkish Economy. Ege Academic Review 2007; 7(1): 153-189. DOI:https://www.researchgate. net/publication/26497969

Cantalejo MJ, Zouaghi F, Perez-Arnedo I. Combined effects of ozone and freeze-drying on the shelf-life of broiler chicken meat. Food Science and Technology 2016; 68: 400-407. DOI:http://dx.doi.org/10.1016/j. Iwt.2015.12.058

Chen F, Mu G, Li C, Peng M, Yang X, Li W. Inhibitory effects of ethyl pyruvate on platelet aggregation and phosphatidylserine exposure. Biochemical and Biophysical Research Communications 2017; 487: 560-566. DOI:http://dx.doi.org/10.1016/j.bbrc.2017.04.087

Del Río E, Panizo-Morán M, Prieto M, Alonso-Calleja C, Capita R. Effect of various chemical decontamination treatments on natural microflora and sensory characteristics of poultry. International Journal of Food Microbiology 2007; 115(3):268-80. DOI:https://doi.org/10.1016/j. ijfoodmicro.2006.10.048

Durak MZ, Churey JJ, Gates M, Sacks GL, Worobo RW. Decontamination of green onions and baby spinach by vaporized ethyl pyruvate. Journal of Food Protection 2012; 75: 1012-1022. DOI:http://jfoodprotection. org/doi/pdf/10.4315/0362-028X.JFP-12-008

Gosselin RE, Hodge HC, Smith RP, Gleason MN. Clinical toxicology of commercial products. Acute poisoning. $4^{\text {th }}$ ed. Baltimore: Williams and Wilkins Press; 1976.

Han Y, Sherman DM, Linton RH, Nielsen SS, Nelson PE. The effects of washing and chlorine dioxide gas on survival and attachment of Escherichia coli 0157:H7 to green pepper surfaces. Food Microbiology 2000; 17: 521-533. DOI:https://doi.org/10.1006/fmic.2000.0343

Hungaro HM, Mendonça RCS, Gouvea DM, Vanetti MCD, Pinto CLO. Use of bacteriophages to reduce Salmonella in chicken skin in comparison with chemical agents. Food Research International 2013; 52:75-81. DOI:https://doi.org/10.1016/j.foodres.2013.02.032

Loretz M, Stephan R, Zweifel C. Antimicrobial activity of decontamination treatments for poultry carcasses: A literature survey. Food Control 2009; 21:791-804. DOl:https://doi.org/10.1016/j.foodcont.2009.11.007

Mahmoud BSM, Linton RHF. Inactivation kinetics of inoculated Escherichia coli 0157:H7 and Salmonella enterica on lettuce by chlorine dioxide gas. Food Microbiology 2008; 25: 244-252. DOl:https://doi. org/10.1016/j.fm.2007.10.015

Nadarajah D, Han JH, Holley RA. Inactivation of Escherichia coli 0157:H7 in packaged ground beef by allyl isothiocyanate. International Journal of Food Microbiology 2005; 99:269-279. DOI:https://doi.org/10.1016/j. ijfoodmicro.2004.08.019

Nierop van W, Duse AG, Marais E, Aithma N, Thothobolo N, Kassel M, Stewart R, Potgieter A, Fernandes B, Galpin JS, Bloomfield SF. Contamination of chicken carcasses in Gauteng, South Africa, by Salmonella, Listeria monocytogenes and Campylobacter. International Journal of Food Microbiology 2005; 99: 1-6. DOl:https://doi:10.1016/j. ijfoodmicro.2004.06.009.

Northcutt J, Smith D, Ingram KD, Hinton JA, Musgrove M. Recovery of bacteria from Broiler carcasses after spray washing with acidified electrolyzed water or sodium hypochlorite solutions. Poultry Science 2007; 86:2239-2244. DOI:https://doi.org/10.1093/ps/86.10.2239

Obaidat MM, Frank JF. Inactivation of Escherichia coli 0157:H7 on the intact and damaged portions of lettuce and spinach leaves by using allyl isothiocyanate, carvacrol, and cinnamaldehyde in vapor phase. Journal of Food Protection 2009a;72:2046-2055. DOl:http://jfoodprotection. org/doi/pdf/10.4315/0362-028X-72.10.2046 
Obaidat MM, Frank JF. Inactivation of Salmonella and Escherichia coli 0157:H7 on sliced and whole tomatoes by allyl isothiocyanate, carvacrol, and cinnamaldehyde in vapor phase. Journal of Food Protection 2009b; 72: 315-324. DOI:http://jfoodprotection.org/doi/ $\mathrm{pdf} / 10.4315 / 0362-028 \mathrm{X}-72.2 .315$

Asif M, Rahman H, Qasim M, Khan TA, Ullah W, Jie Y. Molecular detection and antimicrobial resistance profile of zoonotic Salmonella Enteritidis isolated from broiler chickens in Kohat, Pakistan. Journal of the Chinese Medical Association 2016;80:303-306. Available: http://www.sciencedirect. com/science/article/pii/S1726490117300308?via\%3Dihub

Sagdıc O, Ozturk I, Tornuk F. Inactivation of non-toxigenic and toxigenic Escherichia coli 0157:H7 inoculated on minimally processed tomatoes and cucumbers: Utilization of hydrosols of Lamiaceae spices as natural food sanitizers. Food Control 2013; 30: 7-14. AVAILABLE: http://www. sciencedirect.com/science/article/pii/S095671351200401X

Tornuk F, Durak MZ. A Novel Method for Fresh-Cut Decontamination: Efficiency of Vaporized Ethyl Pyruvate in Reducing Staphylococcus aureus and Escherichia coli 0157:H7 from Fresh Parsley. Journal of Foood Processing and Preservation 2015; 39:1518-1524. DOl:https:// doi.org/10.1111/jfpp. 12377
Tornuk F, Cankurt H, Ozturk I, Sagdic O, Bayram O, Yetim H. Efficacy of various plant hydrosols as natural food sanitizers in reducing Escherichia coli 0157:H7 and Salmonella Typhimurium on fresh cut carrots and apples. International Journal of Food Microbiology 2011; 148: 30 35. Available: http://www.sciencedirect.com/science/article/pii/ S0168160511002455?via\%3Dihu

Bozkurt F, Tornuk F, Toker OS, Karasu S, Arici M, Durak MZ. Effect of vaporized ethyl pyruvate as a novel preservation agent for control of postharvest quality and fungal damage of strawberry and cherry fruits. Food Science and Technology 2015; 65:1044-1049. Available: http:// www.sciencedirect.com/science/article/pii/S0023643815302139

Tseng TF, Liu DC, Chen MT. Evaluation of transglutaminase on the quality of low-salt chicken meat-ball. Meat Science 2000; 55: 427-431. Available: http://www.sciencedirect.com/science/article/pii/S0309174099001722

Vurma M, Pandit RB, Sastry SK, Yousef AE. Inactivation of Escherichia coli 0157:H7 and natural microbiota on spinach leaves using gaseous ozone during vacuum cooling and simulated transportation. Journal of Food Protection 2009; 72:1538-1546. Available: http://jfoodprotection.org/ doi/pdf/10.4315/0362-028X-72.7.1538 